\title{
A Brief Survey on Nonlinear Surface Plasmonics
}

\author{
Yizeng Liang1,a \\ ${ }^{1}$ Zhejiang Normal University, School of Mathematics and Information Engineering, Jinhua, China, 321000 \\ aZSDliangyz@163.com
}

\begin{abstract}
With the rapid development of optical nonlinearities, the study of nonlinear surface plasmonics becomes more popular. Due to the huge potential application value of surface plasmonics, lots of researches in this field become the hotspot. This paper reviewed the history and basic researches of nonlinear plasmonics and focused on several hot topics attracting global attentions. In addition, we introduced the application status of nonlinear surface plasmonics and draw a conclusion. Meanwhile we outlooked the future developments.
\end{abstract}

\section{Introduction}

Over the past decades, nonlinear optical effects have been proved to be useful for many different applications, including nonlinear microscopy [1], ultrafast laser systems [2], optical frequency conversion [3], and all-optical switching [4]. In fact, the optical nonlinearities are inherently weak, because they are governed by photon-photon interaction enabled by materials. Nonlinearities are superlinearly dependent on the electromagnetic field and they can be strengthened in material environment that provide mechanism for field enhancement. An increased effective nonlinear optical response can be achieved through plasmonic effects.

Recently, there has been much interest in studying these phenomena at the nanoscale, particularly in plasmonic structures [5]. Such structures typically incorporate nanoscopic metallic features that enable enhanced nonlinear effects modified by surface plasmon polaritons (SPPs), which are electromagnetic excitations propagating at the interface between a metal and a dielectric and evanescently confined in the perpendicular direction [6]. These electromagnetic surface waves arise via the coupling of the electromagnetic fields of incident light to oscillations of the metal's electron plasma. Because of their capacity to concentrate intense fields over subwavelength scales, SPPs can enhance nonlinear optical processes in metallic nanoparticles as well as at extended metal surfaces by modifying the refractive index near the metal surface which results in significant modifications of the plasmonic resonance [7].

There are many ways of exciting SPPs using either electron beams or photons on planar, rough, or grating surfaces. Historically, electron beam excitation was first used, with optical excitation being introduced in the late 1960s [8]. By far the most versatile technique of exciting SPPs in planar geometries is the method of attenuated total reflection (ATR), which was independently demonstrated by Otto et al. [9].

The nonlinear response of SPPs propagating along different types of plasmonic wave guides has been theoretically investigated by several groups [10-13]. In most of these studies it is assumed that the nonlinearity results from a Kerr-type nonlinear dielectric bounding the metal, while the metal is taken as a linear medium. However, SPPs are intrinsically nonlinear even in the absence of a nonlinear dielectric as the metal exhibit strong and ultrafast third-order nonlinear response at optical wavelengths [14]. Calculation of the intrinsic nonlinear propagation of SPPs supported by two typical plasmonic waveguides: a metallic slab and a semi-infinite metal bounded by linear dielectrics have been shown by Israel [15].

The excitation of a surface plasmon can cause optical signal enhancement which is dipole allowed, including second-harmonic generation (SHG) [16] and third-harmonic generation (THG) [17]. And the SHG and THG depend on the square and cube of the incident intensity. Even the formation of a novel hybrid-vector spatial plasmon-soliton in a Kerr slab embedded in-between metal plates can be predicted and analyzed with a modified NLSE, encompassing hybrid vector field characteristic [18].

This paper is arranged as follows. In Section 2, the basic researches in nonlinear plasmonics are introduced. In Section 3, we reviewed several hot topics attracting global attention. Some latest developments are presented in Section 4. Finally, in Section 5, we draw a conclusion and our expectation in this field.

\section{Basic researches in nonlinear plasmonics}


In 1957, Ritchie, investigated that loss phenomena associated with interaction taking place at metallic surfaces were recorded via the diffraction of electron beams at thin metallic foils, which was then linked with the original work on diffraction gratings in the optical domain in the 1960s. By that time, Kretschmann et al. had achieved the excitation of Sommerfeld's surface waves with visible light using prism coupling in 1968. And then a unified description of all these phenomena in the form of surface plasmon polaritons was established [19].

When light interacts with nanostructures, it can couple to free-electron excitation near the metal surface. The electromagnetic resonances associated with these surface plasmons depend on the details of the nanostructures, opening up opportunities for controlling light confinement on the nanoscale. The resulting strong electromagnetic fields allow weak nonlinear processes, which depend superlinearly on the local field, to be significantly enhanced. In addition to providing enhanced nonlinear effects with ultrafast response times, plasmonic nanostructures allow nonlinear optical components to be scaled down in size [20]. Nonlinear optical effects arise when electronic motion in a strong electromagnetic field cannot be considered harmonic. Expansion of the anharmonicity as power series in the field strength mixes the incident fields and produces new fields that oscillate at the sums and differences of the incident frequencies and can propagate in various directions.

For applications, the most important effects occur at second and third order. The second-order response typically gives rises to wave-mixing effects that lead to frequency conversion, the most common example being second-harmonic generation(SHG), its second harmonic, $2 \omega$. New frequencies arise also from third-order nonlinearities. More importantly, however, the third-order response contains terms at the incident frequencies. This is known as the optical Kerr effect and results in nonlinear modification of the refractive index, allowing all-optical switching and modulation of light. By combining Kerr nonlinearities with optical cavities or other systems with feedback, it is possible outputs. For optical beams with finite transverse size, diffraction and nonlinear effects can balance each other, giving rise to optical solitons.

The plasmonic structure can enhance nonlinear effects in two main ways. First, such structures provide field enhancement near the metal-dielectric interface, associated with the excitation of either SPPs or LSPs. This is characterized by the frequency-dependent local-field factor $L(\omega, r)=\left|E_{\text {loc }}(\omega, r) / E_{0}(\omega)\right|$, where $r$ is the position vector, $E_{l o c}(\omega, r)$ is the local field at frequency $\omega$ associated with the plasmonic excitation, and $E_{0}(\omega, r)$ is the incident field. The resulting strong fields enhance the nonlinear response locally and, when carefully designed, even whole the response is integrated over the whole sample. This concept is equally applicable to increasing the nonlinearity of the metal itself (intrinsic response) or of a material adjacent to the metal (extrinsic response). Second, the plasmonic excitation parameters, including the SPP wavevector and the LSP or metamaterial resonance frequency, are very sensitive to the refractive indices of the metal and the surrounding dielectric. A nonlinear change in the index of either material can therefore significantly modify plasmonic resonances and associated reflection, transmission or absorption of light.

\section{Hot topics in nonlinear plasmonics}

Surface-enhanced nonlinear effects. Surface-enhanced SHG [16] was first studied using electrochemically roughened silver surfaces. The SHG signal was found to be diffuse, but increased by four orders of magnitude relative to a flat reference surface when integrated over all detection directions. The results suggested that the signals are incoherent and enhanced by LSP resonances of the nanoscale surface features.

More-recent work has addressed surface-enhanced SHG from a number of viewpoint. Near-field SHG measurements have been performed using near-field detection and excitation, the latter also correlated with surface topography. Far-field SHG microscopy has emphasized the spatial overlap of the local fundamental and SHG modes. These and additional studies show that the SHG signals depend on the polarization of the fundamental field even if the light is focused down to the achievable resolution of a few hundreds of nanometers.

LSP resonances can also enhance nonlinear processes other than SHG. In particular, surface-enhanced third -harmonic generation has also been observed and its different symmetry rules, relative to those of SHG, discussed.

Enhancement of SHG by propagating SPPs was also demonstrated early on, and SPPs have been used to enhance four-wave mixing (FWM). Very recently, the roles of photonic and plasmonic modes in SHG from metal films have been addressed by momentum-space spectroscopy.

Many scientists believe that the future of signal processing and computing lies not within electronics but in the field of sub-wavelength optics. Controlling light at sub-wavelength scale, forbidden by traditional far-field optics, relies on surface plasmon techniques [21]. SPPs are electromagnetic waves that exist on the surface of metals such as gold or silver. The interaction between the electromagnetic field and the free electrons in the metal helps confine SPPs tightly to the surface. The behavior of this wave can be manipulated by nanostructuring either the surface or a nearby dielectric. By contrast, metallic particles (or nanoscale dielectric voids in a metallic host) can enhance the field by supporting localized plasmonic excitation that create superior field confinement. Both mechanisms can locally control the electromagnetic field, a pre-requisite for the development of such light-based circuity and devices.

In order to directly make plasmonic components, scientists commonly propose two approaches, both based on the modification of the refractive index near the metal surface. The firs uses the electro-optic effect exhibited, for example, by liquid crystal molecules. Here, the refractive index depends on the molecular alignment, 
which can be modulated using an electronic and photonic signals can be transferred, processed, and interconnected in the same metallic circuitry. A second method relies on dielectric materials with a large Kerr nonlinearity, or a refractive index that depends on the intensity of the illuminating light. Wayne Dickson got a result of making plsmonic components with his cooperators, as shown in Fig.1.
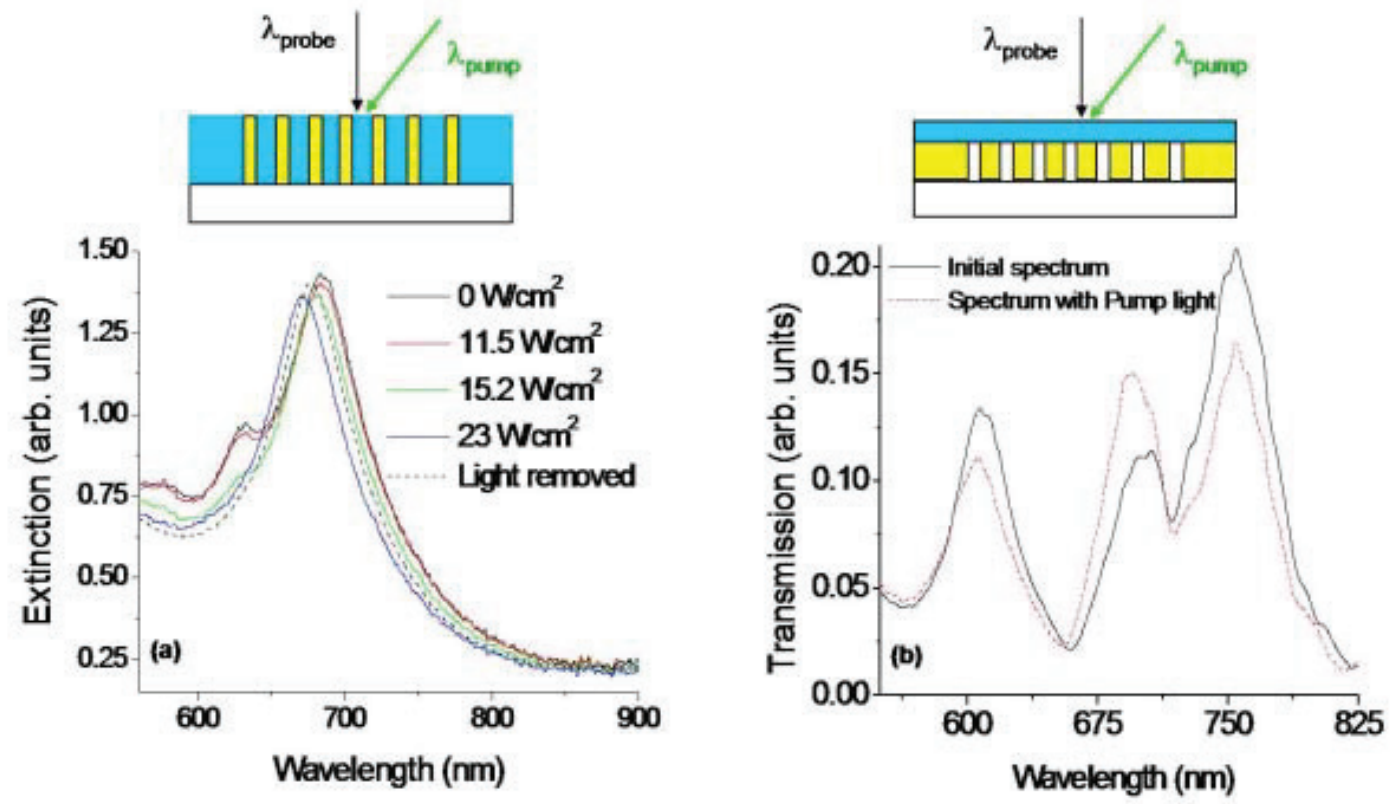

Figure 1. Modulation of transmission by an array of gold nanorads (left) and a whole array in a thin gold film (right). Both were coated with poly-3BCMU (blue in schematic) and pumped with control light at $514 \mathrm{~nm}$.

To modulate and switch SPP signals by optical means in a waveguiding geometry, changes can be included in the real or imaginary part of the permittivity by control light, and these changes can occur in the metal or the adjacent dielectric. Light-induced absorption modulation has explored with the intrinsic metal ( $\mathrm{Au}$ and $\mathrm{Ga}$ ) nonlinearity [22-23] as well as the dielectric component in a plasmonic waveguide [24].

The intrinsic nonlinearity of gold is related to the interband excitation of non-equilibrium electrons and results in small changes in the real part of the permittivity but in more considerable changes in the imaginary part. The associated relaxation time is on the picosecond scale [25], and terahertz-rate direct modulation of SPP signals in plasmonic gold waveguides is thus possible. The variation of SPP modes via modification of their wave. To convert this to intensity modulation. Various phase-sensitive configuration, including Mach-Zehnder interferometers and Waveguide-ring resonators, are needed.

Periodically structured metal films and surfaces, the so-called plasmonic or surface plasmon polaritonic crystals (SPPCs), provide considerable versatility for tailoring the wavelength-dependent plasmonic resonances and the electromagnetic field enhancement in devices a few tens of wavelengths in size [26]. SPPCs have optical properties analogous to those of two-dimensional photonic crystals. The spectral positions of their resonances, their dispersion, and the phase and group velocities of SPP Bloch modes can be engineered by controlling the size and shape of the unit cell and its period. In particular, relatively flat SPP modes with low group velocity are possible, resulting in the high field enhancement beneficial for high effective nonlinearity. These effects have been demonstrated using the nonlinearity of gold gratings to control SPP excitation at speeds determined by the subpicosecond response time of the gold nonlinearity.

\section{Latest developments in nonlinear plasmonics}

The method of calculating the nonlinear propagation of surface plasmon polaritons in one-dimensional planar waveguides consisting of a metal slab or a semi-infinite metal bounded by linear dielectrics, starting with an assumed third-order nonlinearity that characterizes the nonlinear response of the metal has been demonstrated by Israel. With this approach he model the self-phase-modulation of surface plasmon polaritons. Expressions relating the complex nonlinear parameter of these surface wave with the third-order nonlinear susceptibility of the metal are provided. We present and discuss results pertaining to the self-phase-modulation of the symmetric and antisymmetric surface plasmon polaritons supported by a thin gold slab in vacuum and of the surface plasmon polaritons supported by the single gold-vacuum interface [15].

The expression for the complex nonlinear parameter that characterizes the self-phase-modulation in term of the structural properties of the wave guide, and use it to construct a differential equation that describes the evolution of the SPP's complex amplitude can be derived. 
Solving this differential equation provides us with a self-consistent way to estimate the nonlinear phase and nonlinear loss experienced by the SPP upon propagation. Israel De Leon and his cooperators got the result of estimating the nonlinear phase and nonlinear loss experienced by the SPP upon propagation as shown in Fig.2.
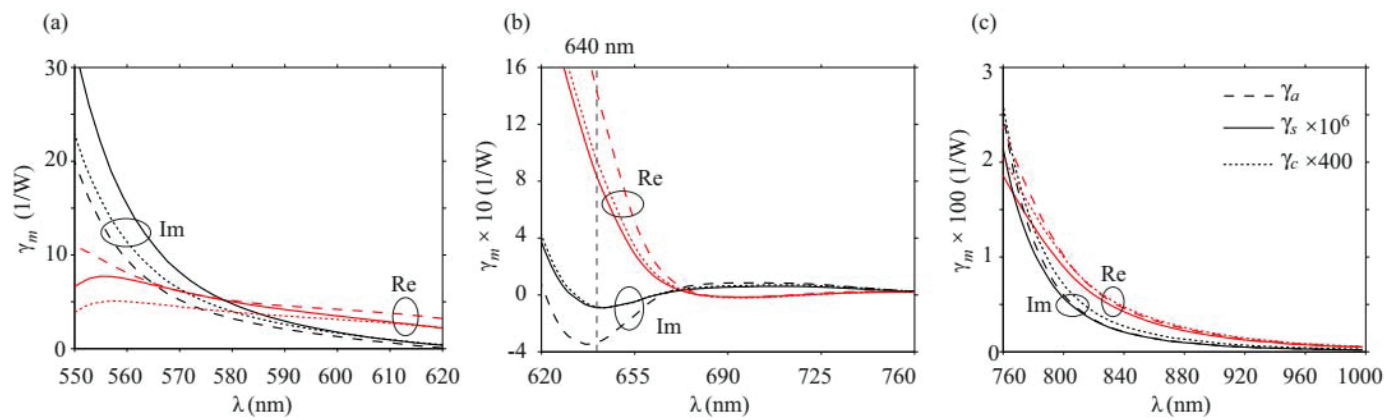

Figure 2. Complex nonlinear parameter of the various SPP modes $m=(s, a, c)$, calculated over three different wavelength ranges. The values of $\gamma_{s}$ and $\gamma_{c}$ have been multiplied by a factor of $10^{6}$ and 400 , respectively, before plotting. (Color online) Plasmon-Soliton

The derivation of a hybrid vector NLSE describing slab confined TM modes which are self-trapped in the free (in-plan) dimension by the Kerr effect [27]. A nonlinear slab sandwiched between metal layers supports a hybrid plasmon-soliton, and exhibits high confinement with lower effective beam size than the conventional diffraction limit-although practically the soliton self-trapping dimension is not reduced below the diffraction limit with available nonlinear material. Assisted by the transverse plasmonic effect, the self-trapping dimension of the plasmon-soliton was substantially compressed when reducing the slab width. The practical limitation of the plasmon-soliton size reduction is determined by available nonlinear materials and metal loss. A novel dispersion characteristic-the nonlinear index dispersion, exhibits saturation at small gap width-while the linear effective index keeps increasing indefinitely.

The field of nanophotonics is finding myriad applications in information technology, health care, lighting, sensing and national security. The scientific community's interest in this area was recently sparked with the discovery of enhanced light transmission through nanoholes in an array or with surrounding surface corrugations. Even with a hole far below the cutoff of the enhanced fields, due to the resonant SPP excitation, the amount of light scattered through the holes was several times larger than expected for an isolated subwavelength hole [28]. The excitation and visualization of ultrafast dynamics of SPP modes can be achieved in experiments by coupling the optical field into and out of the metallic film using a 2D array of nanoholes etched in the metallic film on a dielectric substrate. By measuring the transmittance for a number of samples with various periods, one can gain a better understanding of the modal structure of SPP fields. The spectra are dominated by asymmetric, Fano-type lineshapes at the various SPP excitation conditions. There are two components to this transmitted field: one that passes through the small, subwavelength holes directly, and one that is a product of the excited surface mode. The interference between these two components results in various maxima and minima.

\section{Conclusion}

In this article, we reviewed the basic researches of nonlinear of plasmonic, which is still a young research field. And its basic concepts have been demonstrated in a wide variety of ways. Also we reviewed the hot topics and the latest developments in nonlinear plasmonics. The role of plasmonics in nonlinear optics is threefold. First, by enhancing the effective nonlinearity, plasmonic nanostructures contribute to conventional photonics by allowing nonlinear effects to be utilized with reduced optical power. Second, they make it possible to scale down nonlinear components in size, which is important for the development of integrable photonic devices. Third, the response time of plasmonic excitations is ultrafast, allowing optical signals to be manipulated on femtosecond timescales. The field of plasmonics offers several research opportunities. To fulfill the promise offered by plasmonics, more research needs to be done in these areas. Some of the challenges that face plasmonics research in the coming years are as follows: (i) demonstrate optical frequency subwavelength metallic wired circuits with a propagation loss that is comparable to conventional optical waveguides. (ii) Develop deep subwavelength plasmonic nanolithography over large surfaces.

\section{Reference}

1. Y. Wang, C.-Y. Lin, A. Nikolaenko, V. Raghunathan, and E. O. Potma, Adv. Opt. Photon. 3, 1 (2011).

2. G. Cerullo and S. De Silvestri, Rev. Sci. Instrum. 74, 1 (2003).

3. M. Fejer, phys. Today 47, 25 (1994).

4. D. Cotter, R. Manning, K. Blow, A. Ellis, and A. Kelly, Science 286, 1523 (1999). 
5. M. Kauranen and A. V. Zayats, Nat. Photon. 6, 737 (2012).

6. H. Raether, Surface Plasmons on Smooth and Rough Surfaces and on Gratings (Springer, Berlin, 1988).

7. J. A. Schuller, E. S. Barnard, W. Cai, Y. C. Jun, J. S. White, and M. L. Brongersma, Nat. Mater. 9, 193 (2010).

8. H. RAETHER, physics of Thin Films 9 (1977) 145.

9. A. OTTO, Z. phys. 216 (1968) 398 and Phys. Stat. Solidi. 26 (1968) 199.

10. D. Mihalache, G. I. Stegeman, C. T. Seation, E. M. Wright, R. Zanoni, A. D. Boardman, and T. Twardowski, Opt. Lett. 12, 187 (1987).

11. H.Yin, C. Xu, and P. M. Hui, Appl. Phys. Lett. 94, $221102(2009)$

12. J.-H. Huang, R. Chang, p.-T. Leung, and D. P. Tsai, Opt. Commun. 282, 1412 (2009).

13. A. R. Davoyan, I. V. Shadrivov, and Y. S. Kivshar, Opt. Express 16, 21209 (2008).

14. F. Hache, D. Ricard, C. Flytzanis, and U. Kreibig, Appl. Phys. A 47, 347 (1988).

15. I. D. Leon, J. E. Sipe, and R. W. Boyd, Phys. Rev. A 89, 013855 (2014).

16. R. M. Corn, M. Romagnoli, M. D. Levenson and M. R. Philpott, Chemical. Phys. Lett. Vol 106, Number 12 (1984)

17. T. Y. F. Tsang, Opt. Lett. Vol 21. No 4 (1996).

18. E. Feigenbaum and M. Orenstein, Opt. Lett. 32, 674 (2007).

19. S. A. Maier, Plasmonics: Fundamentals and Applications, 2006.

20. M. Kauranen and A. V. Zayats, Nature. Photo. Vol 6, (2012)

21. W. Dickson, A. Krasavin, G. Wurtz, and A. Zayats, Newsroom, 10.1117.

22. Rotenberg, N. Betz, M. \& van Driel, H. M. Ultrafast all-optical coupling of light to surface plasmon polaritons on plain metal surfaces. Phys. Rev. Lett. $105,017402(2010)$

23. Krasavin, A. V. \& Zheludev, N. I. Active plasmonics: controlling signals in $\mathrm{Au} / \mathrm{Ga}$ waveguide using nanoscale structural transformations. Appl. Phys. Lett. 84,1416-1418 (2004).

24. Pacific, D. Lezec, H. J.\& Atwater, H. A. All-optical modulation by plasmonic excitation of $\mathrm{CdSe}$ quantum dots. Nature Photon. 1, 402-406 (2007).

25. Baida, $H$. et al. Ulfast nonlinear optical response of a single gold nanorod near its surface plasmon resonance. Phys. Rev. Lett. 107, 057402 (2011).

26. Wurtz, G. A. \& Zayats, A. V. Nonlinear surface plamon polaritonic crystals. Laser Photon. Rev. 2, 125-135 (2008).

27. E. Feigenbaum and M. Orenstein, Opt. Lett. 32, 674 (2007).

28. Y. Fainman, K. Tetz, R. Rokitski and P. Lin, Optics \& Photonics. 17, 24-29 (2006). 